\author{
Antonio Gangemi ${ }^{1 *}$, Theresa Dunham ${ }^{2}$ \\ Federico Gheza ${ }^{1}$, Gianmarco Contino', \\ Pier Cristoforo Giulianotti ${ }^{1}$ \\ ${ }^{1}$ University of Illinois at Chicago, Division of \\ General, Minimally Invasive, Robotic Surgery, \\ Department of Surgery, USA \\ ${ }^{2}$ University of Illinois at Chicago, College of \\ Medicine, USA
}

Dates: Received: 29 April, 2016; Accepted: 11 May, 2016; Published: 12 May, 2016

*Corresponding author: Dr. Antonio Gangemi, University of Illinois at Chicago, Division of General, Minimally Invasive, Robotic Surgery, Department of Surgery, 840 S. Wood Street, Code 958, Suite 435E, Chicago, 60612, , USA, Tel: 312-355-2494; Fax: 312355-3722; E-mail: agangemii@gmail.com

www.peertechz.com

ISSN: 2455-2968

Keywords: Robotic surgery training curriculum; Surgical residency robotic simulation education; Virtual reality robotic surgery
Research Article

\section{Robotic Training in General Surgery Residency: How Early Can We Begin?}

\section{Abstract}

Background: The increasing demand for robotics in general surgery has prompted academic institutions to train general surgery residents toward the acquisition of basic robotic skills. Our current robotic training curriculum begins in the PGY-3 year and is based on the use of surgical simulators in a risk-free environment, in which each resident must show proficiency prior to advancing to training on an animate model as PGY-4. Our unpublished data on the curriculum indicates that PGY-3s required additional remediation training on the robotic simulator, suggesting room for improvement in our teaching paradigm [8]. Because of resident duty hour restrictions, we could not provide remediation by simply increasing the number of training sessions. We therefore decided to investigate an alternative strategy of shifting the training to an earlier time point in general surgical residency during PGY-1 and PGY-2 years. To explore the feasibility of a new curriculum, we undertook a pilot study to investigate the willingness of residents in their PGY-1 and PGY-2 years to begin robotic training on the robotic simulator, the $\mathrm{dV}$-Trainer ( $\mathrm{dV}$ - T). We also wanted to see if even minimal early exposure to the dV-T would help overcome residents' initial diffidence in using the daVinci Surgical System (DaVss).

Methods: Ten general surgery residents (seven PGY-1s and three PGY-2s) with no prior exposure to robotic training were randomly distributed into MIMIC (MIM G) and daVinci (DaV G) groups. The MIM G subjects answered a post-exposure questionnaire about their overall experience with the robotic training. The five MIM G subjects performed five basic skills exercises on the $\mathrm{dV}$-T simulator prior to executing the same exercises on the DaVss, while the five DaV G subjects performed the same exercises only on the DaVss. Two blinded robotic proctors scored each subject's performance on the DaVss.

Results: All MIM G subjects found their overall experience constructive and viewed the dV-T as useful in preparing them to complete subsequent tasks on the DaVss. The MIM G subjects also performed better than the DaV G $(p=0.32)$ subjects in operation of the da-Vss, although statistical significance could not be achieved. Given the small sample size, statistical significant was unlikely.

Conclusions: The subjective perception of the $\mathrm{dV}-\mathrm{T}$ experience was strongly positive, as the residents enjoyed the experience and seemed to be open to the possibility of introducing some robotic training with the robotic simulator earlier in their career. We attribute the fact that MIM G residents performed better with the DaVss than the DaV G residents to the value of minimal exposure to dV-T as a way to overcome the discomfort of using the DaVss for the first time.

\section{Introduction}

The use of robotics in medicine has increased from 25,000 per year in 2005 to 450,000 per year in 2012 [1]. In particular, surgical robots accounted for over $60 \%$ of the largest share in 2014 , due to the increasing demand for minimally invasive surgeries and the growing use of surgical robotic equipment in hospitals [1]. In fact, the global market for robotic systems is expected to reach 17.9 billion USD by the year 2022, with an estimated growth rate of $12.7 \%$ from 2014 to 2020 [1]. Expanding upon the experience of a group of general surgeons who had previously pioneered the development and implementation of robotic surgery in their field, the use of this technology in general surgery is now catching up to other robotic sub-specialties [2]. A growing number of general surgery procedures, ranging from bariatric procedures to colon resections, are being performed robotically $[3,4]$. Naturally, the trend of more robotic general surgery cases is accompanied by a requirement for more robotically trained general surgeons. This increasing need for robotic training poses multiple challenges, such as limited hands-on teaching during live surgery, the expense of animal model robotic training, and the resource- and time-consuming need to train not only surgical residents and fellows, but also seasoned and experienced laparoscopic attending physicians.

The robotic platform is complex, unique and requires dedicated training. The use of surgical simulators in a training environment is ideal because there are no risks imposed upon patients. We use the $\mathrm{dV}$-Trainer $(\mathrm{dV}-\mathrm{T})$ surgical simulator for the training of our residents because its user interface, content, and construct validity have been demonstrated by multiple studies and it closely recreates a tridimensional field [5-7].

At our institution, we have implemented a standardized robotic training surgical curriculum in which general surgery PGY-3 residents complete five one-hour training sessions and one final examination prior to advancing to training on an animate model, in which they must complete six one-hour training labs and one final examination. In our curriculum, $\mathrm{dV}$ - $\mathrm{T}$ training does not commence until the PGY-3 year under the assumption that robotics training is a complex activity that requires at least some degree of prior experience with minimally-invasive surgical techniques. We felt that PGY- 
1 and PGY-2 residents, who are are just beginning their careers as general surgery trainees, might perceive such additional training as overwhelming,

However, we found that all PGY-3 residents required at least one remediation session before achieving basic robotic competency in the stimulated environment, delaying their advancement to the use of the DaVss on the porcine model [8]. However, due to the 80-hour week duty restrictions in general surgery residency, we struggled to add more training sessions throughout the academic year. Instead, we considered the possibility that PGY-3 may not necessarily be the optimal time to introduce robotics and training and we pursued an alternative strategy of shifting robotics training to an earlier time point in residency. We implemented a pilot study examining PGY1 and PGY-2 residents' willingness to participate in robotic training with the $\mathrm{dV}-\mathrm{T}$ simulator. By comparing robotic surgery performance parameters between trainees who participated in simulator training to those who did not, we examined the quantitative and qualitative impact of the simulation training at an early stage of surgical residency on these individuals' subsequent performance on and perception of the DaVss.

\section{Methods and Materials \\ Curriculum}

In our proposed early introduction to robotics, PGY-1 and PGY2 residents performed five basic skills exercises on the $\mathrm{dV}-\mathrm{T}$ and/or the DaVss, depending on the study group. The five exercises included overview of controls with special emphasis on the use of robotic gimbals, camera and clutch pedals, camera targeting, pick and place, and ring and rope walk.

\section{Subjects}

The research activities conducted at our surgical skills laboratory involving training and surgical simulation are covered with approval from our Institutional Review Board.

We recruited ten general surgery residents, seven PGY-1s and three PGY-2s, without prior experience in robotic surgery, and randomly distributed them into two groups. Five subjects were assigned to the MIMIC group (MIM G); these subjects performed the five exercises that comprised the curriculum on the $\mathrm{dV}$-T simulator, and subsequently executed the same tasks in the daVss dry lab. The other five subjects were assigned to the daVinci group ( $\mathrm{DaV} G$ ); these subjects performed the five exercises on the DaVss without prior exposure to the $\mathrm{dV}$-T simulator.

\section{Qualitative assessment}

The MIM G residents were queried about their perception of their $\mathrm{dV}$-T experience. They completed a six-question Self-Assessment Questionnaire about MIMC Experience, in which each question was answered on a five-point analog scale (Figure 1).

\section{Quantitative assessment}

The dV-T software assessed the residents' performances using its built-in MScore system, which factors comprehensive metrics and
1. How closely do you think the virtual surgical field of the simulator represents the DaVinci surgical field?

2. Did the use of the simulator facilitate your confidence with the use of the DaVinci's clutch and camera pedals?

3. Did the simulator facilitate the use of wrist range of motion rather than your entire arm while performing the surgical tasks on the DaVinci?

4. Did the simulator help you to avoid unnecessary collisions between instruments while performing task on the DaVinci?

5. Overall how helpful was the simulator in improving your confidence when using the DaVinci the first time?

6. Do you think that additional exposure to the simulator would further improve your

Figure 1: Self-Assessment Questionnaire about MIMIC Experience (Analog Scale 0-5).

experiential data from more than 100 surgeons in order to provide a parameter on which to grade users of the simulator. The assessment was designed so that the resident had to achieve a proficiency score of $\geq 80 \%$ in a task set before proceeding to the next task set.

The performance of both the MIM G and DaV G groups on the DaVss was assessed by two general surgery proctors, both formally trained in robotics and minimally invasive surgery and both with at least one year of experience teaching in the robotics lab. The proctors were blinded to whether the subjects had prior training on the $\mathrm{dV}-\mathrm{T}$ simulator. The two raters were trained on the use of the assessment tool, which was a rubric extrapolated from the validated Global Evaluative Assessment of Robotic Skills (GEARS) tool [9], (Figure 2). This tool addressed the subjects' depth perception, efficiency, and other skills. We also included a final question addressing the potential of the trainee as a future robotic trainee.

\section{Statistical analysis}

For the GEARS tool quantitative assessment, the mean values for the dependent variables were compared with one-way repeated measures ANOVA. Bonferroni corrections were performed when factors were significant. All statistical analyses were performed using the SPSS. The significance level was set at $\mathrm{p} \leq 0.05$.

\section{Results \\ Qualitative assessment}

The MIM G subjects reported a positive perception of their experience with the $\mathrm{dV}$-T prior to operation of DaVss. $80 \%$ of the residents scored their overall perception of the $\mathrm{dV}-\mathrm{T}$ as extremely helpful. These residents reported that the virtual field of the MIMIC simulator was very close $(80 \%)$ or extremely close $(20 \%)$ to the daVss field, and $80 \%$ of them reported that the $\mathrm{dV}$-T was either extremely $(60 \%)$ or very helpful $(20 \%)$ in improving their confidence with the use of the DaVss pedals. $80 \%$ of the interviewed subjects reported that the $\mathrm{dV}$ - T was extremely helpful in preventing unnecessary collisions while using the DaVss for the first time. Interestingly, only $20 \%$ of them believed that no additional exposure would be necessary to further improve their confidence while approaching the DaVss, suggesting that most residents believed more practice would be beneficial. 


\begin{tabular}{|c|c|c|c|c|}
\hline Depth perception & & & & \\
\hline 1 & 2 & 3 & 4 & 5 \\
\hline $\begin{array}{l}\text { Constantly overshoots } \\
\text { target, wide swings, } \\
\text { slow to correct }\end{array}$ & & $\begin{array}{l}\text { Some overshooting or } \\
\text { missing of target, but } \\
\text { quick to correct }\end{array}$ & & $\begin{array}{l}\text { Accurately directs } \\
\text { instruments in the } \\
\text { correct plane to target }\end{array}$ \\
\hline
\end{tabular}

\section{Bimanual dexterity}

\begin{tabular}{|c|c|c|c|c|}
\hline 1 & 2 & 3 & 4 & 5 \\
\hline $\begin{array}{l}\text { Uses only one hand, } \\
\text { ignores nondominant } \\
\text { hand, poor coordination }\end{array}$ & & $\begin{array}{l}\text { Uses both hands, but } \\
\text { does not optimize } \\
\text { interaction between } \\
\text { hands }\end{array}$ & & $\begin{array}{l}\text { Expertly uses both } \\
\text { hands in a } \\
\text { complementary way to } \\
\text { provide best exposure }\end{array}$ \\
\hline \multicolumn{5}{|l|}{ Efficiency } \\
\hline 1 & 2 & 3 & 4 & 5 \\
\hline $\begin{array}{l}\text { Inefficient efforts; } \\
\text { many uncertain } \\
\text { movements; constantly } \\
\text { changing focus or } \\
\text { persisting without }\end{array}$ & & $\begin{array}{l}\text { Slow, but planned } \\
\text { movements are } \\
\text { reasonably organized }\end{array}$ & & $\begin{array}{l}\text { Confident, efficient and } \\
\text { safe conduct, maintains } \\
\text { focus on task, fluid } \\
\text { progression }\end{array}$ \\
\hline
\end{tabular}

progress

\section{Force sensitivity}

\begin{tabular}{|c|c|c|c|c|}
\hline 1 & 2 & 3 & 4 & 5 \\
\hline $\begin{array}{l}\text { Rough moves, tears } \\
\text { tissue, injures nearby } \\
\text { structures, poor } \\
\text { control, frequent } \\
\text { suture breakage }\end{array}$ & & $\begin{array}{l}\text { Handles tissues } \\
\text { reasonably well, minor } \\
\text { trauma to adjacent } \\
\text { tissue, rare suture } \\
\text { breakage }\end{array}$ & & $\begin{array}{l}\text { Applies appropriate } \\
\text { tension, negligible } \\
\text { injury to adjacent } \\
\text { structures, no suture } \\
\text { breakage }\end{array}$ \\
\hline \multicolumn{5}{|l|}{ Autonomy } \\
\hline 1 & 2 & 3 & 4 & 5 \\
\hline $\begin{array}{l}\text { Unable to complete } \\
\text { entire task, even with } \\
\text { verbal guidance }\end{array}$ & & $\begin{array}{l}\text { Able to complete task } \\
\text { safely with moderate } \\
\text { guidance }\end{array}$ & & $\begin{array}{l}\text { Able to complete task } \\
\text { independently without } \\
\text { prompting }\end{array}$ \\
\hline \multicolumn{5}{|l|}{ Robotic control } \\
\hline 1 & 2 & 3 & 4 & 5 \\
\hline $\begin{array}{l}\text { Consistently does not } \\
\text { optimize view, hand } \\
\text { position, or repeated } \\
\text { collisions even with } \\
\text { guidance }\end{array}$ & & $\begin{array}{l}\text { View is sometimes not } \\
\text { optimal. Occasionally } \\
\text { needs to relocate } \\
\text { arms. Occasional } \\
\text { collisions and } \\
\text { obstruction of } \\
\text { assistant. }\end{array}$ & & $\begin{array}{l}\text { Controls camera and } \\
\text { hand position optimally } \\
\text { and independently. } \\
\text { Minimal collisions or } \\
\text { obstruction of assistant }\end{array}$ \\
\hline
\end{tabular}

Figure 2: Global Evaluative Assessment of Robotic Skill (GEARS).

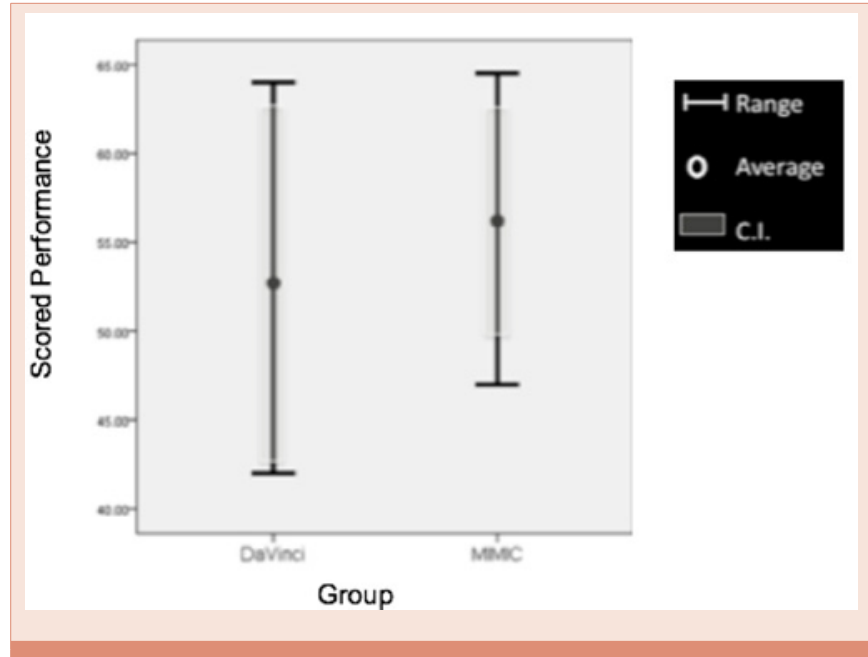

Figure 3: Assessment of Trainees on DaVss.

\section{Quantitative assessment}

When comparing the performance of both MIM G and DaV $G$ subjects on the DaVss, a positive trend was noted relating the experience of robotic simulation with $\mathrm{dV}$ - $\mathrm{T}$ to subsequent achievement when operating the DaVss (Figure 3). The MIM G subjects, on average, scored higher when using the DaVss. Although this did not reach statistical significance $(\mathrm{p}=0.32)$, the small sample size likely represented the main limiting factor.

\section{Discussion}

The gap between demand for robotic surgical procedures and availability for robotic training is growing [10]. An investigative survey found that although more than $96 \%$ of participating residents reported that their institutions had a surgical robotic system, only $18 \%$ of these individuals reported experience with operating the robotic console [11]. $60 \%$ of the residents also indicated that they received no education or training prior to their first robotic case [11]. 
We believe it is important that academic institutions begin to train general surgery residents toward the acquisition of at least the basic robotic skills in a risk-free environment prior to exposing them to real life patients. This conviction is in accordance to the approach that has already been largely validated in other medical and non-medical fields, such as civil and military aviation [12].

Based on time restrictions, we currently offer our PGY-3 and PGY-4 residents only five or six one-hour sessions for robotic simulator training, which are scheduled on Saturdays in order to prevent schedule conflict with clinical duties, throughout the year. However, our unpublished data has suggested that this number of sessions is insufficient in achieving proficiency as documented by the number of remediation session required by our residents [8]. To investigate whether introducing robotic training earlier in residents' career is a feasible solution, we assessed the responses of PGY-1 and PYG-2 residents to first time exposure to robotic training simulation. It is worth noting that our intent was not to prove face validity of the robotic simulator, but instead we meant to sample intern and junior residents' perception of the robotic experience earlier in their surgical career than had been done with our prior training curriculum. We were pleased by the enthusiasm of our novice residents, who expressed an overall strongly positive perception of the virtual reality robotic training. These results are promising and perhaps indicate that novices have a more open-minded approach to robotic training than we had previously assumed.

In addition, quantitative assessment showed a positive trend relating minimal exposure to the $\mathrm{dV}$-T and more successful operation of the Da-Vss. We do not believe that this positive correlation can be explained by the formal benefit of a single exposure to the $\mathrm{dV}-\mathrm{T}$. Instead, we interpret this result to mean that only minimal exposure to the $\mathrm{dV}$-T might be sufficient to overcome the initial diffidence related to the first time use of the DaVss. We hope that this study can provide guidance to other academic institutions interested in either implementing or fine-tuning an existing robotic training curriculum. We also hope that this study can prime growing interest among other general surgery residency programs in the conversation regarding robotic surgery and the future it holds.

Our study was mainly limited by its small sample size, which does not allow us to draw definitive conclusions about our results. Furthermore, the Self-Assessment Questionnaire was created 'ad hoc' to sample our novice residents' about their initial experience with robotic surgery training, and we acknowledge that this is not a validated survey. However, we had to make a strategic decision regarding our curriculum for the upcoming academic year, so it was important for us to gather residents' feedback in an efficient way. We would have liked to adopt a previously validated survey, but to our knowledge, there are no similar initiatives available in literature.

\section{Conclusions}

PGY-1 and PGY-2 residents' subjective perception of the dV-T experience was overall strongly favorable. The results also showed a higher level of performance by first-time users of the DaVss after minimal exposure to $\mathrm{dV}$-T, as compared to subjects without previous exposure to dV-T. The small study sample size did not allow us to draw any definitive conclusions, but our results suggest potential feasibility and benefit in the implementation of a robotic simulation curriculum early (PGY-1 and PYG-2 years) in the training of general surgery residents.

\section{References}

1. Grand View Research (2015) Medical Robotic Systems Market Analysis by Product (Surgical, Orthopedic, Laparoscopy, Neurological, Rehabilitation, Assistive, Prosthetics, Orthotics, Steerable, Therapeutic, Exoskeleton, NonInvasive, Hospital/Pharmacy, Telemedicine, I.V, Pharmacy, Emergency Response Robotic Systems) and Segment Forecasts To 2022.

2. Giulianotti PC, Coratti A, Angelini M, Sbrana F, Cecconi S, et al. (2003) Robotics in general surgery: personal experience in a large community hospital. Arch Surg 138: 777-784.

3. Park SY, Choi GS, Park JS, Kim HJ, Choi WH (2012) Robot-assisted right colectomy with lymphadenectomy and intracorporeal anastomosis for colon cancer: technical considerations. Surg Laparosc Endosc Percutan Tech 22: e271-276.

4. Ayloo SM, Buchs NC, Addeo P, Giulianotti PC (2011) Laparoendoscopic single-site adjustable gastric banding: technical considerations. Surg Laparosc Endosc Percutan Tech 21: 295-300.

5. Lallas CD, Davis JW, Members of The Society of Urologic Robotic Surgeons (2012) Robotic surgery training with commercially available simulation systems in 2011: a current review and practice pattern survey from the society of urologic robotic surgeons. J Endourol 26: 283-293.

6. Kang SG, Ryu BJ, Yang KS, Ko YH, Cho S, et al. (2015) An effective repetitive training schedule to achieve skill proficiency using a novel robotic virtual reality simulator. J Surg Educ 72: 369-376.

7. Egi H, Hattori M, Tokunaga M, Suzuki T, Kawaguchi K, et al. (2013) Face, content and concurrent validity of the Mimic ${ }^{\circledR} \mathrm{dV}$-Trainer for robot- assisted endoscopic surgery: a prospective study. Eur Surg Res 50: 292-300.

8. Gangemi A (2015) The changing role of the surgical skills laboratory in the MIS training of general surgery residents: from a "cherry picking" approach to a standardized, proficiency-based curriculum. ACS AEI Consortium Quarterly Winter 20155

9. Goh AC, Goldfarb DW, Sander JC, Miles BJ, Dunkin BJ (2012) Global Evaluative Assessment of Robotic Skills: Validation of a Clinical Assessment Tool to Measure Robotic Surgical Skills. J Urol 187: 247-252.

10. Buchs NC (2012) Training in Robotic General Surgery: The Next Challenge. Adv Robot Autom 1: e104.

11. Farivar BS, Flannagan M, Leitman IM (2015) General surgery residents' perception of robot-assisted procedures during surgical training. J Surg Educ 72: $235-242$

12. Lateef $F$ (2010) Simulation-based learning: Just like the real thing. J Emerg Trauma Shock 3: 348-352.

Copyright: (c) 2016 Gangemi A, et al. This is an open-access article distributed under the terms of the Creative Commons Attribution License, which permits unrestricted use, distribution, and reproduction in any medium, provided the original author and source are credited. 